\title{
KERNFORSCHUNGSZENTRUM
}

\author{
KARLSRUHE
}

Abteilung Reaktorbetrieb

Untersuchungen an einem bestrahlten FR2-Brennelement

W. Dienst, H. -L. Krautwedel

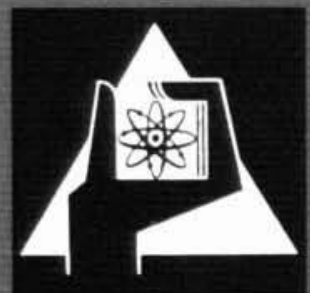

OESELLSCHAFT FUR KERNFORS CHUNG M. B. H. 


\section{Untersuchungen an einem bestrahlten FR2-Brennelement}

Von Wolfgang Dienst und Hans Ludwig Krautwedel in Karlsruhe 


\title{
Untersuchungen an einem bestrahlten FR2-Brennelement
}

\author{
Von Wolfgang Dienst und Hans Ludwig Krautwedel in Karlsruhe
}

(Aus der Abteilung Reaktorbetrieb im Kernforschungszentrum Karlsruhe)

Der im Forschungsreaktor FR2 in Karlsruhe eingesetzte Kernbrennstoffelementtyp aus einem Natururanstab mit Aluminiumhülle wurde vor Aufnahme des Vollastbetriebes am FR2 geeigneten Prüfungen und Bewährungsproben unterworfen. Zu diesen gehörten Werkstoffuntersuchungen an unbestrahlten Brennelementen ${ }^{1}$ ) und vor allem Bestrahlungsversuche an zwei FR2-Brennelementen im NRX in Chalk River. An dem im Bestrahlungsversuch stärker belasteten Brennelement wurden eingehende Untersuchungen durchgeführt, die Aufschluß über die durch die Bestrahlung eingetretenen Eigenschaftsänderungen des metallischen Uranbrennstoffes geben sollten.

Über das Verhalten von metallischem Uran als Kernbrennstoff liegen in der Literatur zahlreiche Angaben vor, die teilweise durch die hier erhaltenen Ergebnisse erweitert werden. Im wesentlichen handelt es sich dabei um folgende Literaturangaben für reines metallisches Uran:

Die Dichte nimmt durch Bestrahlung bei mäßigen Temperaturen und Abbränden $\left(\mathrm{T}<300{ }^{\circ} \mathrm{C}\right.$, Abbrand $0,5 \%$ ) um 0,3 bis $0,5 \%$ je $0,1 \%$ Abbrand $\left.a b^{2}\right)^{\text {bis } 6}$ ). Bei Bestrahlungstemperaturen oberhalb von etwa $400{ }^{\circ} \mathrm{C}$ steigt die Dichteabnahme durch die Ansammlung von Spaltgasen in Poren (,swelling“) auf einige $\%$ je $0,1 \%$ Abbrand $\left.{ }^{2}\right)^{5}$ ) bis 7 ). Die thermische Leitfähigkeit nimmt durch $0,1 \%$ Abbrand um 10 bis $20 \%$, die elektrische Leitfähigkeit um etwa $\left.10 \% \mathrm{ab}^{2}\right)^{3}$ ). Die Härte steigt durch $0,1 \%$ Abbrand bei mäßigen Temperaturen $\left(\mathrm{T}<300{ }^{\circ} \mathrm{C}\right)$ um 100 bis 200 Vickershärteeinheiten, durch $0,05 \%$ Abbrand um 70 bis 120 Vickershärteeinheiten an ${ }^{2}{ }^{\text {bis } 8}$ ). Die Verformbarkeit geht schon durch $0,02 \%$ Abbrand fast vollständig verloren $(\delta<1 \%)$. Eine Erholung ist innerhalb des a-Phasenbereiches $\left(\mathrm{T}<650^{\circ} \mathrm{C}\right)$ nicht möglich $\left.\left.{ }^{3}\right)^{9}\right)$. Dadurch ergibt sich eine ausgeprägte Anfälligkeit für die Bildung von Mikrorissen durch die stark anisotrope thermische Ausdehnung des Urans im Verlauf von Temperaturwechseln $\left.{ }^{3}\right)^{10}$ ).

Im Gefüge des Urans zeigen sich nach Bestrahlung zahlreiche feine Zwillinge, die von Verformungszwillingen deutlich verschieden sind. Nach Abbränden über $0,02 \%$ erscheinen diese Zwillinge gekrümmt oder geschert $\left.{ }^{8}\right)^{11}$ ). Nach relativ hohem Abbrand von etwa

1) D. C. Minty, R. F. Hills und B. R. Butcher, AERE-Report M 699 (1960).

2) A. A. B a uer, M. S. Far a s und V. W. S horhok, REIC-Report 29 (1963).

3) S. H. B u s h, HW-Report 51444 (1957).

$\left.{ }^{4}\right)$ J. H. Kittel und S. H. P a ine, ANL-Report 5539 (1957)

5) S. F. Pugh, J. Nucl. Mat. 4 (1961) 177.

6) C. A 11 a in, CEA.Report 1110 (1959).

7) G. W. Green wo od , AERE-Report R 2864 (1959).

8) J. B I o ch, J. P. Mustelier, P. Bussy und J. B I i n, CEA-Report 987 (1958).

9) D. S h a w, Nucl. Engineering 5 (1960) 214.

10) R. C. B e $11 \mathrm{a} \mathrm{m} \mathrm{y} \mathrm{,} \mathrm{Proc.} \mathrm{Venice} \mathrm{Conf.} \mathrm{Irradiation} \mathrm{Effects}$ on Solids (1962), Radiation Damage in Reactor Materials. IAEA, Wien (1963) S. 59.

11) J. Bloch und J. B ourgues, CEA-Report 1190 (1959).

$0,1 \%$ entspricht das Gefügebild dem von stark verformtem Uran. Das Röntgenfeinstrukturdiagramm zeigt Linienverbreitungen bis zu $50 \%{ }^{12}$ ).

\section{Grundlagen des Untersuchungsprogramms}

Der Brennstoffteil des FR2-Brennelementes umfaßt einen 2,16 m langen zylindrischen Stab mit $32 \mathrm{~mm} \mathrm{Dmr}$. aus natürlichem Uran, dem zur Kornfeinung 0,4 Gew.. $\%$ $\mathrm{Nb}$ zulegiert sind. Niob ist im $\alpha$-Uran bei niedrigen
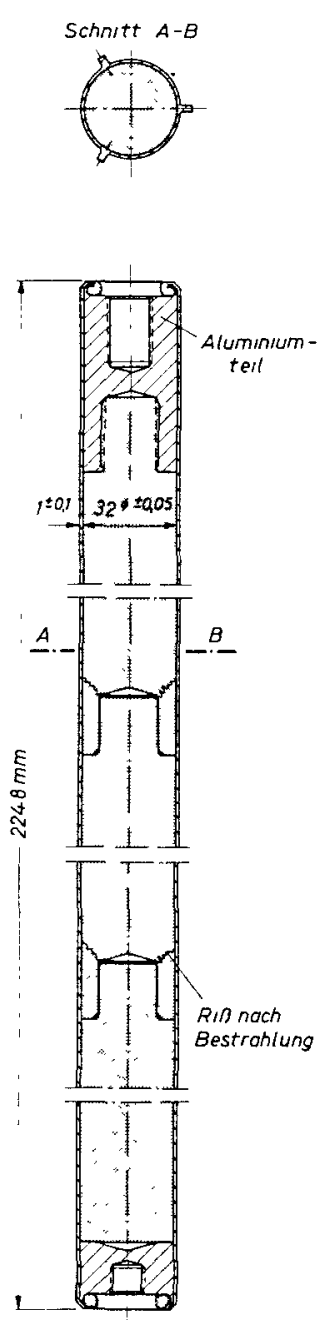

Bild 1. Skizze des FR2. Brennelementes (in der Zeichnung ist der Riß angedeutet, der während der Bestrahlung an den Preßsitzen entstand)
Temperaturen kaum löslich und liegt in feinen Korngrenzenausscheidungen vor. Die Brennstoffhülle wird durch ein Reinaluminiumrohr $(99,5 \mathrm{Gew} .0 \%$ von $1 \mathrm{~mm}$ Wanddicke gebildet, das durch zwei Schichten aus Zinn und Zink mit dem Uranbrennstoffstab verlötet ist. Die Lötschicht soll gleichzeitig als Wärmekontakt und Diffusionsbarriere zwischen Uran und Aluminium dienen ${ }^{13}$ ). Zur Zentrierung im Kühlwasserführungsrohr ist das Aluminiumhüllrohr mit drei Längsrippen versehen.

Die Brennstoffstäbe wurden aus fertigungstechnischen Gründen aus drei gleichlangen Teilstücken hergestellt, die durch Preßsitz miteinander verbunden sind(Bild 1 ). Der Erzeugung eines Kristallgefüges mit regellos orientierten, feinen Körnern im Uranbrennstoff diente eine Glühbehandlung mit nachfolgender Abschreckung aus dem $\gamma$-Phasengebiet. Durch Wärmebehandlung und mechanische Bearbeitung hervorgerufene innere Spannungen wurden durch eine abschließende Glühung im $\alpha$ Phasengebiet beseitigt ${ }^{14}$ ).

Das untersuchte Brennelement wurde im NRX bei einem maximalen thermischen Neutronenfluß von 3,2 . $10^{13} \mathrm{n} / \mathrm{cm}^{2} \mathrm{~s}$ (entsprechend $1500 \mathrm{~W} / \mathrm{cm}$ ) bis zu einem

${ }^{12}$ ) S. H. Pa in e und J. H. Kitte l, Proc. First Geneva Conf. (1955). UN, New York 7 (1956) P/745, S. 445.

13) G. S c h n ei d e r, Metall 15 (1961) 675.

14) A. B o et $\mathrm{tch}$ er, G. Matz und G. Schneider, Proc. Second Geneva Conf. (1958). UN, Genf 6 (1958) P/1004, S. 449 . 
mittleren Abbrand von $900 \mathrm{MWd} / \mathrm{t}$ bestrahlt. Der maximale Abbrand im Brennstoffstab betrug 1100 $\mathrm{MWd} / \mathrm{t}$ (entsprechend $0,12 \%$ ). Die Flußwerte sind jeweils über den Brennstoffstabquerschnitt gemittelt. Die maximale Brennstoffhüllentemperatur war auf etwa $90^{\circ} \mathrm{C}$ begrenzt. Die axiale Neutronenflußverteilung am Brennstoffstab während des Bestrahlungsversuches ist in Bild 2 in grober Näherung durch eine Sinusfunktion wiedergegeben, die auch als genäherte Darstellung der Abbrandverteilung angesehen werden kann. Der aus der Neutronenflußverteilung berechnete Verlauf der Zentraltemperatur des Brennstoffstabes bei der höchsten Belastung ist ebenfalls aus Bild $2 \mathrm{zu}$ entnehmen. Die höchste Temperatur betrug danach $535^{\circ} \mathrm{C}$.

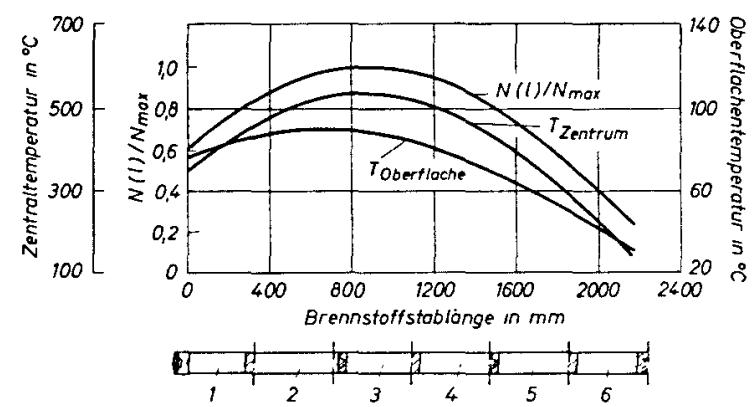

Bild 2. Relative spezifische Wärmeleistung, Zentraltemperatur und Oberfiächentemperatur des bestrahlten Brennelementes bei einem maximalen Neutronenfluß von $3,2 \cdot 10^{13} \mathrm{n} / \mathrm{cm}^{2} \mathrm{~s}$ (unten schematisch das Brennelement, in dem die Probenentnahmebereiche angedeutet sind)

Längenmessungen und Röntgenaufnahmen des Brennstoffstabes im Verlauf des Bestrahlungsversuchs zeigten im Bereich seiner Teilstücke keine Längen- oder Durchmesseränderungen, die wesentlich über die Fehlergrenzen der Meßverfahren hinausgingen. Dadurch wurde die Voraussetzung bestätigt, daß das Kristallgefüge des Uranbrennstoffes praktisch texturfrei war. Dagegen waren im Bereich der beiden Preßsitze Aufbeulungen am Uranstab zu erkennen, die sich über den gesamten Umfang und recht genau über die axiale Länge des Zapfens im Preßsitz erstreckten.

\section{Bearbeitungs- und Untersuchungsverfahren}

Probenent n h m e. Das bestrahlte Brennelement wurde bereits am NRX in sechs Abschnitte von je etwa $38 \mathrm{~cm}$ Länge zerschnitten, die vom unteren zum oberen Ende des Brennelementes fortschreitend die Bezeichnungen 1 bis 6 erhielten. Bild 2 zeigt diejenigen Teile die. ser Abschnitte, aus denen die Proben für die hier beschriebenen Untersuchungen entnommen wurden. Sie sollten zur Vereinfachung der Probenentnahme möglichst an den Enden der Abschnitte liegen, neben Bereichen höchsten Neutronenflusses und Abbrands auch solche wesentlich niedrigerer Belastung erfassen und vor allem die von vornherein als besonders schadenanfällig erkannten Preßsitze des Brennstoffstabes beinhalten.

Die etwa fünfzig Einzelproben wurden aus den Brennstoffstababschnitten 1 bis 6 mit einer Trennschleifmaschine entnommen, die zu diesem Zweck provisorisch in einer heißen Zelle des Karlsruher Isotopenlaboratoriums installiert worden war (Bild 3). Der Trennvorgang erfolgte mit einer schnell laufenden Karborundscheibe unter Wasser in einem Plexiglasbecken. Mit der. selben Vorrichtung wurden entsprechende Proben aus einem unbestrahlten FR2-Brennelemert entnommen, die für die notwendigen Vergleichsuntersuchungen an unbestrahltem Material herangezogen wurden.

Für die Untersuchung der radioaktiven Proben stand eine gasdichte Bleizelle zur Verfügung ${ }^{15}$ ). Sie enthält

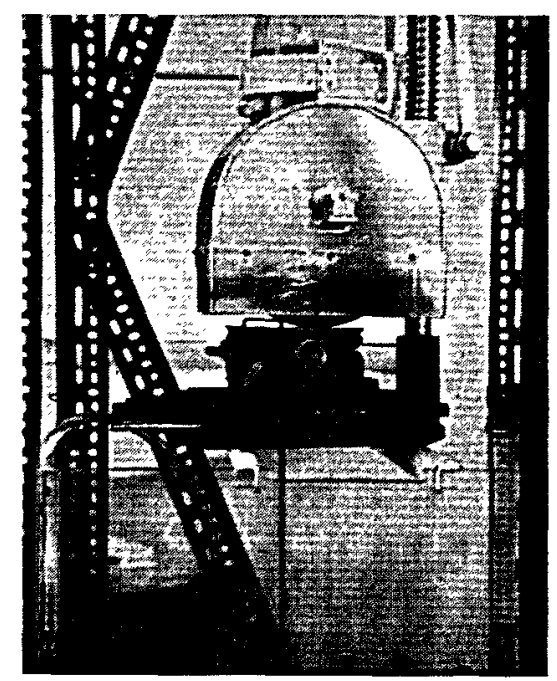

Bild 3. Unterwassertrennmaschine mit eingespanntem Brennelementabschnitt in der heißen Zelle

Einrichtungen für metallographische Arbeiten und für Messungen der Härte, Dichte und elektrischen Leitfähigkeit (Bild 4).

Mikroskopische Untersuchung. Zur mikroskopischen Untersuchung bestimmte Proben vurden mit einer Einbettpresse in Bakelit eingebettet. Dem verwendeten Bakelitpulver waren 6 Gew.- $\%$ Graphitpulver beigemischt, um die Einbettmasse für die später folgende elektrolytische Behandlung der Schliffprobe elektrisch leitend zu machen. Die eingebetteten Proben wurden auf wasserfestem SiC-Papier (Körnungen 240, 320, $400,600)$ naß geschliffen und anschließend mit Diamantpaste (Körnungen 6 und $1 \mu$ ) auf Vibrationspoliergeräten vorpoliert. Vor der mikroskopischen Betrachtung im polarisierten Licht folgte eine elektrolytische

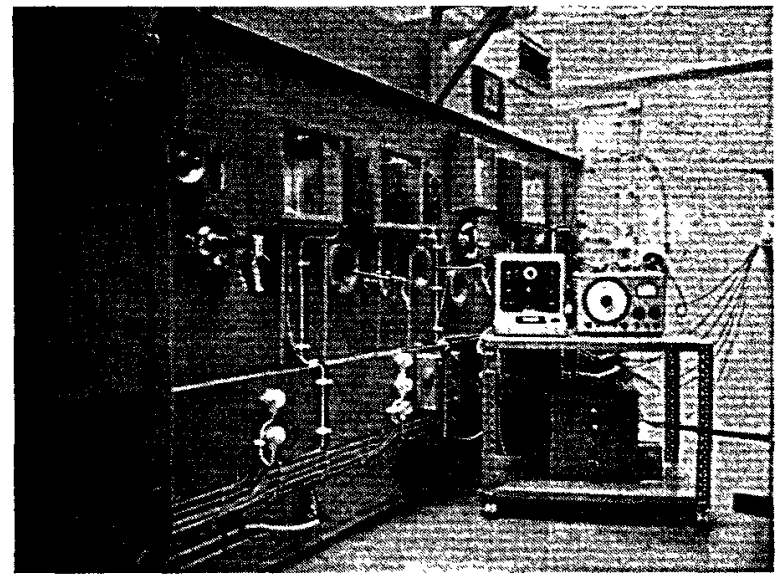

Bild 4. Bedienungsseite der metallographischen Zelle

Polierbehandlung mit einer Lösung von 5 Teilen Phosphorsäure, 5 Teilen Äthylenglykol und 8 Teilen Äthylalkohol. Die Polierspannung betrug etwa $40 \mathrm{~V}$, die Polierzeit 30 s. Zwischen den einzelnen Arbeitsgängen wurden die Proben in einem Ultraschallreinigungsbad mit Alkohol gesäubert und mit einem Föhn getrocknet.

15) H. L. Kra utwedel, Kemtedmik T (1965) 26. 
Für die unbestrahlten Vergleichsproben wurde das elektrolytische Polieren meist durch eine Ätzpolierbehandlung ersetzt, die auf die radioaktiven Proben mangels geeigneter Einrichtung der verfügbaren Untersuchungszelle nicht anwendbar war. Das Ätzpolieren weist einige Vorteile auf: es erfaßt die gesamte Schlifffläche und führt nicht zur Bildung von Ätzgrübchen und Auflösung von Verunreinigungen. Die Schliffprobe ist nicht nur für die Betrachtung des Gefüges im polarisierten Licht, sondern auch für die Untersuchung von Gefügeeinschlüssen bei Hellfeldbeleuchtung geeignet. Das Ätzpolieren erfolgte auf Mikrotuch mit einer Aufschlämmung von Tonerde 1 in einer $\mathrm{CrO}_{3}-\mathrm{HNO}_{3}-\mathrm{H}_{2} \mathrm{O}$-Lösung.

Die mikroskopischen Untersuchungen wurden für die radioaktiven Proben mit einem fernbedienbaren Metallmikroskop Reichert-Telatom durchgeführt, für die unbestrahlten Vergleichsproben mit dem entsprechenden normalen Metallmikroskop Reichert-MeF.

Physikalische Messungen. Für die Härtemessungen wurde ein Härteprüfer Wolpert-Diatestor verwendet, der zur Prüfung der radioaktiven Proben mit den notwendigen Fernbedienungsvorrichtungen ausgestattet war. Im allgemeinen wurden Vickershärtewerte bestimmt (HV 50, für unbestrahlte Proben HV 30), die nach Zweckmäßigkeit durch Brinellhärtewerte ergänzt wurden.

Zur Messung der elektrischen Leitfähigkeit stand ein Förster-Sigmatest-Meßgerät zur Verfügung, das nach dem Prinzip der Wirbelstromerregung arbeitet. Ein derartiges Gerät ist (bei mäßiger relativer Meßgenauigkeit von etwa $1 \%$ ) zur Anwendung an radioaktiven Proben recht gut geeignet, weil nur die kleine Induktionsmeßspule in die Untersuchungszelle eingeführt zu werden braucht. Die geschliffenen Proben können dann ohne besondere Halterung lose auf den Meßkopf aufgelegt werden. Die erhaltenen Leitfähigkeitswerte sollten bei dieser Untersuchung lediglich einen indirekten Nachweis relativ grober Ânderungen der thermischen Leitfähigkeit des Uranbrennstoffes durch Bestrahlung gestatten. Da für Uran das Wiedemann-Franzsche Gesetz befriedigend erfüllt ist, ist die Annahme berechtigt, daß auch die Wärmeleitung in Uran überwiegend durch Leitungselektronen erfolgt und damit durch Strahlenschäden im Kristallgitter gleiche relative Verminderungen erleidet wie die elektrische Leitfähigkeit ${ }^{16}$ ). Da das Kristallgefüge der untersuchten Proben praktisch texturfrei war, brauchte keine Störung dieses Zusammenhanges durch Anisotropieeinflüsse befürchtet zu werden.

Die Dichtebestimmungen wurden nach dem Auftriebsverfahren mit einer Mettler-Analysenwaage und einer fernbedienbaren Hebevorrichtung für den Flüssigkeitsbehälter durchgeführt. Als Auftriebsflüssigkeit wurde Toluol verwendet. Die Temperatur der Flüssigkeit wurde nach jeder Wägung auf $0,01{ }^{\circ} \mathrm{C}$ genau gemessen, die Meßwerte wurden zur Dichteberechnung gemittelt. Meßanordnung und -verfahren gestatteten Dichtebestimmungen mit einer relativen Genauigkeit von $2 \cdot 10^{-4}$. Diese Genauigkeit wurde für jede Probe durch drei voneinander unabhängige Messungen kontrolliert. Alle zur Dichtebestimmung herangezogenen Proben wurden vor den Wägungen einer Ultraschallreinigung in Alkohol unterworfen. Die radioaktiven Proben aus dem bestrahl. ten Brennelement wurden vor der Ultraschallreinigung

16) A. D. B r a i l s f o r d und K. G. M a jor, J. Nucl. Mat. 8 (1963) 241. in halbkonzentrierter Salpetersäure und halbkonzentrierter Salzsäure gebeizt und in Wasser gespült, um die die Meßergebnisse verfälschende starke oberflächliche Oxidschicht zu entfernen.

Für Längenmessungen, die im Verlauf der Untersuchung notwendig werden konnten, standen zwei fernbedienbare Mikrometerschrauben mit Meßbereichen von 0 bis $25 \mathrm{~mm}$ und 25 bis $50 \mathrm{~mm}^{\circ}$ zur Verfügung. Einige Röntgenfeinstrukturdiagramme wurden mit einem normalen Siemens-Zählrohrgoniometer aufgenommen, das für die Messungen an einer radioaktiven Probe lediglich mit einer einfachen fernbedienbaren Probenhalterung versehen war. Die aus dem bestrahlten Brennelement entnommene Probe wurde so klein gehalten, daß ihre eigene $\gamma$-Strahlung (etwa $10 \mathrm{mrem} / \mathrm{h}$ in $1 \mathrm{~m} \mathrm{Abstand)}$ bei Verwendung eines Proportionalzählrohres mit Impulshöhendiskriminator ohne besondere Abschirmung des Zählrohres in ausreichendem Maße von der $\mathrm{Cu}-\mathrm{Ka}$ Meßstrahlung getrennt werden konnte. Zur Kristallgitterkonstantenbestimmung wurde ein Goldeichpräparat herangezogen.

\section{Ergebnisse der Untersuchung von Bestrahlungs- effekten \\ Uranbrennst of $f$}

Gefügeschädigung. Bild 5 zeigt das Urangefüge eines unbestrahlten Brennstoffstabes. Es läßt die regellose Kornorientierung und die Feinkörnigkeit des Urans erkennen. Die genaue Bestimmung der Korngröße war schwierig, weil die Orientierungsunterschiede zwischen benachbarten Körnern oft nicht für eine einwandfreie Identifizierung der Korngrenzen ausreichen. Die durchgeführten Messungen ergaben eine durchschnittliche Korngröße von etwa $25 \mu$. Eine genaue Betrachtung der Gefüge läßt in den Urankörnern zahlreiche feine Ausscheidungs- und Einschlußteilchen erkennen, die sich im Bereich der in Verformungsrichtung gestreckten Lunker zu Zeilen häufen (Bild 6). Bei diesen Verunreinigungen handelt es sich hauptsächlich um die isomorphen Verbindungen UO, UC und UN und ihre Mischkristalle. $\mathrm{U}(\mathrm{O}, \mathrm{C}, \mathrm{N})$ liegt in rechteckig erscheinenden hellen Kristallen vor, UC in rechteckig erscheinenden dunklen Kristallen und UO in Teilchen globularer Form ${ }^{17}$ ).

Bild 7 zeigt das Urangefüge im Abschnitt 1 (Verhältnis des Neutronenflusses zum maximalen Fluß etwa 0,82 ) des bestrahlten Brennstoffstabes von außen nach innen fortschreitend in etwa 14, 10 und $0 \mathrm{~mm}$ Abstand von der Stabachse. In diesen Bereichen herrschten bei höchster Belastung während des Bestrahlungsversuches Temperaturen von etwa 170,300 und $450^{\circ} \mathrm{C}$. Die Proben aus den Abschnitten 3 und 4 (Neutronenflußverhältnis etwa 0,98) zeigten bei einer etwas höheren Zentraltemperatur von $525^{\circ} \mathrm{C}$ sehr ähnliche Gefüge.

Bild 7 a läßt erkennen, daß bei niedriger Temperatur das ursprüngliche Gefüge völlig zerstört worden ist. Zwillingslamellen sind nicht mehr zu sehen. Korngrenzen können kaum noch verfolgt werden. Die ursprünglichen Körner sind in viele, wesentlich kleinere und kristallographisch sehr verschieden orientierte Bereiche aufgeteilt. Die niedrige Temperatur dieser Brennstabzone während des Bestrahlungsversuches und die Gefügeausbildung (im besonderen die großen Orientierungsunterschiede) im Bereich der ursprünglichen

17) K. E. G. Meredit h und M. B. W a l d ro $n$, J. Inst. Met. 87 (1958) 311. 
Körner schließen plastische Verformungen durch Temperaturwechsel als Ursache der Gefügeschädigung weitgehend aus. Die Zerstörung des Gefüges wird durch das Bestrahlungswachstum der Urankristallite verursacht. Im vielkristallinen Gefüge behindern sich die unter Bestrahlung wachsenden Körner gegenseitig. So kommt es zu einer beträchtlichen Verformung (zunächst durch Zwillingsbildung bis zur Erschöpfung dieser Ver- das Wirken eines mit der Bestrahlungsschädigung gleichzeitig ablaufenden Erholungsprozesses. Andernfalls wäre eine weitgehende Auflösung der kristallo. graphischen Ordnung und eine Gitteraufweitung durch Spaltatome und aus ihren Kristallgitterpositionen verlagerte Uranatome zu erwarten. Allerdings zeigt das Röntgenfeinstrukturdiagramm eine starke Verbreiterung der einzelnen Kristallgitterreflexe. Die Halbwerts-

$140 \times$

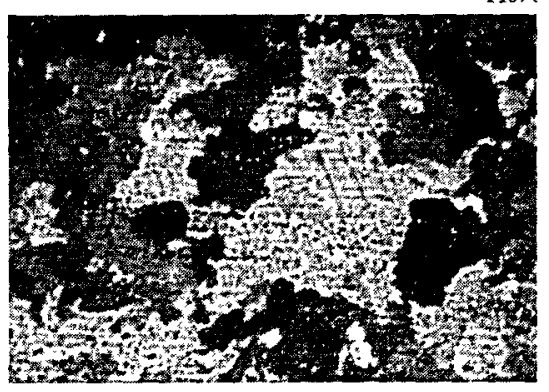

Bild 5 a. Schliff parallel zur Preßrichtung

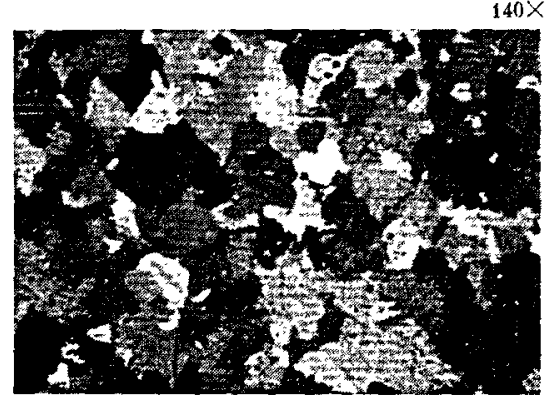

Bild $5 \mathrm{~b}$. Schliff quer zur Preßrichtung

Bild 5 a und b. Gefüge eines unbestrahlten FR2-Brennelementes (elektrolytisch poliert, polarisiertes Licht)

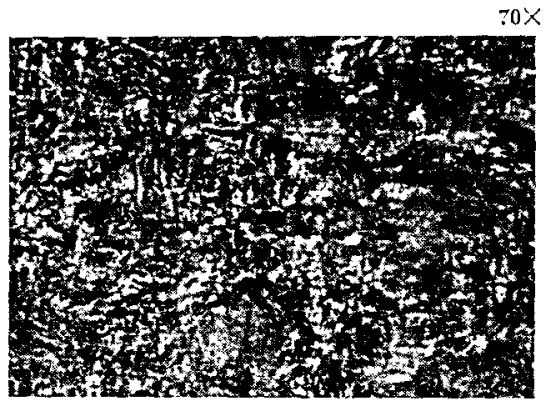

Bild 7 a. Schliff parallel zur Preß richtung, Außenzone

$70 \times$

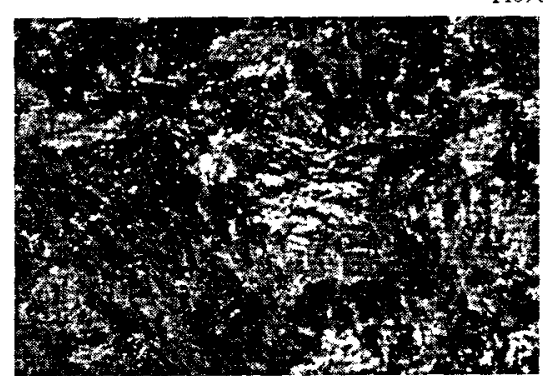

Bild 7 b. Schliff parallel zur PreBrichtung, mittlere Zone
$385 \times$

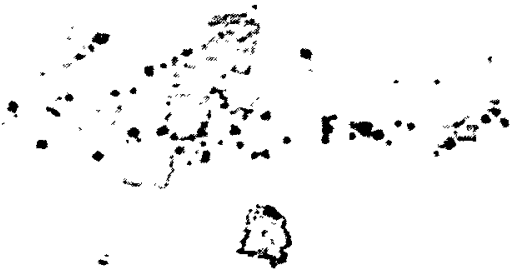

Bild 6. Verunreinigungen in einem unbestrahlten Brennelementabschnitt, Schliff parallel zur Preßrichtung (ätzpoliert, Hellfeld)

Bild 7 a bis c. Gefüge des bestrahlten Brennelementes im Abschnitt 1 (polarisiertes Licht)

formungsmöglichkeit), die schließlich mit steigendem Abbrand zu einer völligen Zerstörung des ursprünglichen Gefüges führt. Bei höheren Bestrahlungstemperaturen entstehen Gefügeschäden vorzugsweise durch plastische Verformungen infolge von Temperaturwechseln. Wie Bild $7 \mathrm{~b}$ und $\mathrm{c}$ zeigt, wird dieser Prozeß jedoch von gleichzeitig ablaufenden Ausheilungsvorgängen begleitet, so daß im zentralen Bereich des Brennstoffstabes eine wesentlich geringere Gefügeschädigung $\mathrm{zu}$ beobachten ist.

Proben aus dem Abschnitt 6 zeigten nad dem in diesem Bereich wesentlich niedrigeren Abbrand (Neutronenflußverhältnis etwa 0,55 ) eine deutlich geringere $\mathrm{Ge}$ fügeschädigung bei niedrigen Bestrahlungstemperaturen. Bild 8 gibt einen Gefügeausschnitt wieder, der eine Bestrahlungstemperatur von höchstens $200^{\circ} \mathrm{C}$ $(r>10 \mathrm{~mm})$ aufgewiesen haben kann und daher etwa mit dem in Bild 7 a abgebildeten Gefüge höheren $\mathrm{Ab}$ brandes zu vergleichen ist. Die Probe aus Abschnitt 6 läßt noch deutlich vollständige Korngrenzen erkennen.

Röntgenfeinstrukturaufnahmen der Probe mit dem in Bild 7 a wiedergegebenen stark geschädigten Gefüge bewiesen, daß die kristallographische Ordnung in den Kristalliten mit innerhalb der relativen Meßgenauigkeit von $5 \cdot 10^{-4}$ unveränderten Kristallgitterkonstanten aufrechterhalten geblieben ist. Dieses Ergebnis bestätigt breiten der zur Auswertung herangezogenen (112)-, (114) - und (154) -Reflexe waren durch Bestrahlung jeweils um etwa $100 \%$ vergrößert (von $(A 2 \theta)_{1 \mathrm{Q}}=0,12$, 0,20 und $0,48^{\circ}$ auf $0,25,0.40$ und $\left.0,90^{\circ}\right)$. Bild 9

$\overline{z 0 x}$

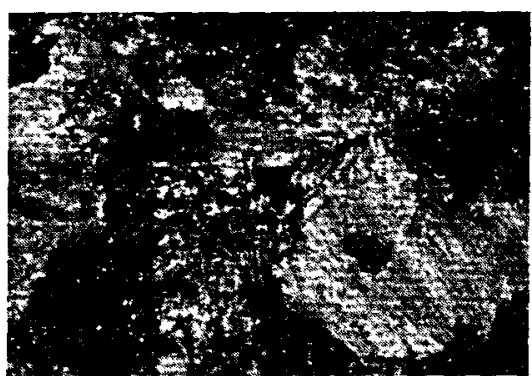

Bild 8. Gefüge des bestrahlten Brennelementes. Schliff quer zur Preßrichtung. Außenzone in Abschnitt 6 (polarisiertes Licht)

zeigt das registrierte Profil des (154)-Reflexes der bestrahlten Probe aus der Randzone des Brennstoffstab. abschnittes 1 und einer Probe entsprechender Lage aus dem unbestrahlten Brennelement, deren Gefüge dem in Bild 5 a abgebildeten sehr ähnlich war. Die Reflexverbreiterung wird durch die angesichts des Gefügebildes (Bild $\tau$ a) beinahe selbstverständlichen Kristallgitterverzerrungen, durch unvollständige Erholung gestörter 
Gitterbereiche und wahrscheinlich auch durch Einlagerung von Spaltproduktatomen im Urangitter verursacht.

Eine Auflösung oder Vermehrung der im unbestrahlten Uranbrennstoff nachgewiesenen zahlreichen Verunreinigungsteilchen, eine Neubildung von Poren oder Anzeichen einer Materialermüdung durch Bildung von Mikrorissen konnten im Gefügebild der bestrahlten Proben nicht nachgewiesen werden. Dagegen waren zeilenförmige Anordnungen von Poren und Verunreini-
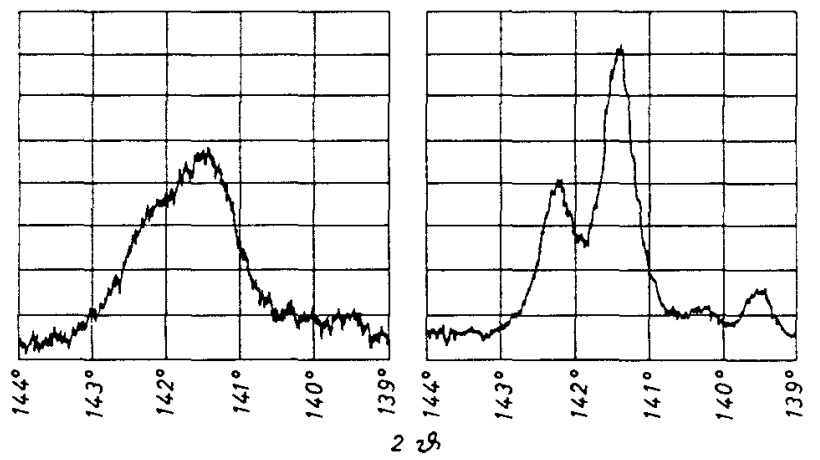

Bild 9. (154)-Reflex von Proben des unbestrahlten (rechts) und bestrahlten Brennelements (links)

T a b ell e 1. Vickershärte (HV 50) des bestrahlten Brennstoffstabes

\begin{tabular}{|c|cccc|}
\hline $\begin{array}{c}\text { Abschnitt } \\
\text { Nr. }\end{array}$ & $\begin{array}{c}\text { Uran- } \\
\text { abbrand } \\
\text { in } \%\end{array}$ & $\begin{array}{c}\text { Maximale } \\
\text { Zentral- } \\
\text { temperatur } \\
\text { in }{ }^{\circ} \mathrm{C}\end{array}$ & Härte HV 50 in $\mathrm{kp} / \mathrm{mm}^{2}$ \\
\hline \hline 1 & 0,10 & 450 & 415 & 270 \\
3 & 0,12 & 530 & 420 & 285 \\
4 & 0,12 & 520 & 430 & 270 \\
5 & 0,10 & 440 & 415 & 265 \\
6 & 0,065 & 300 & 395 & 324 \\
\hline
\end{tabular}

Tabelle 2. Dichte der bestrahlten Brennstoffstababschnitte

\begin{tabular}{|c|cccc|}
\hline $\begin{array}{c}\text { Abschnitt } \\
\text { Nr. }\end{array}$ & $\begin{array}{c}\text { Uran- } \\
\text { abbrand } \\
\text { in } \% \%\end{array}$ & $\begin{array}{c}\text { Maximale } \\
\text { Zentral- } \\
\text { temperatur } \\
\text { in }{ }^{\circ} \mathrm{C}\end{array}$ & $\begin{array}{c}\text { Dichte in } \mathrm{g} / \mathrm{cm}^{\mathbf{3}} \\
\text { Randzone }\end{array}$ & Zentralzone \\
\hline 1 & 0,10 & 450 & 18,853 & 18,788 \\
3 & 0,12 & 530 & 18,839 & - \\
4 & 0,12 & 520 & 18,839 & 18,754 \\
5 & 0,10 & 440 & 18,836 & - \\
6 & 0,065 & 300 & 18,858 & 18,803 \\
\hline
\end{tabular}

Tabelle 3. Elektrische Leitfähigkeit des bestrahlten Brennstoffstabes

\begin{tabular}{|c|c|c|c|c|}
\hline \multirow{2}{*}{$\begin{array}{c}\text { Abschnitt } \\
\mathrm{Nr} \text {. }\end{array}$} & \multirow{2}{*}{$\begin{array}{l}\text { Uran- } \\
\text { abbrand } \\
\text { in } \%\end{array}$} & \multirow{2}{*}{$\begin{array}{l}\text { Maximale } \\
\text { Zentral. } \\
\text { temperatur } \\
\text { in }{ }^{\circ} \mathrm{C}\end{array}$} & \multicolumn{2}{|c|}{$\begin{array}{l}\text { Elektrische Leitfähigkeit } \\
\text { in } \mathrm{m} / \Omega \mathrm{mm}^{2}\end{array}$} \\
\hline & & & Randzone & Zentralzone \\
\hline$-\overline{-}=-$ & $=-$ & & & \\
\hline 1 & 0,10 & 450 & 3,19 & 3,14 \\
\hline 4 & 0,12 & 520 & 3,20 & 3,18 \\
\hline 6 & 0,065 & 300 & 3,18 & 3,15 \\
\hline
\end{tabular}

gungen (Bild 5 a und 6) im wesentlichen nur noch im oberen, während des Bestrahlungsversuches schwächer belasteten Teil des Brennstoffstabes (Abschnitt 6) zu erkennen. Diese Beobachtung dürfte auf plastische Verformungen durch Temperaturwechselspannungen zurückzuführen sein, die in den Brennstoffstabbereichen hoher Bestrahlungstemperatur bei großen Änderungen des Neutronenflusses durch die anisotrope thermische Ausdehnung der Urankörner hervorgerufen wurden.

Änderung der Härte. Die mechanische Werkstoffprüfung an mehreren Uranproben aus dem unbestrahlten Brennelement ergab bei Raumtemperatur eine Härte von $\mathrm{HV} 30$ (und $\mathrm{HB}(30 / 2,5))=(270 \pm 10)$ $\mathrm{kp} / \mathrm{mm}^{2}$. Die Streckgrenze, Zugfestigkeit und Bruchdehnung lagen bei $\sigma_{0,2}=43 \mathrm{kp} / \mathrm{mm}^{2}, \sigma_{\mathrm{B}}=88,5 \mathrm{kp} / \mathrm{mm}^{2}$, $\delta=6,5 \%$. Bei $300{ }^{\circ} \mathrm{C}$ ergaben sich Werte von $\sigma_{0,2}$ $=31,5 \mathrm{kp} / \mathrm{mm}^{2}, \sigma_{\mathrm{B}}=49,5 \mathrm{kp} / \mathrm{mm}^{2}$ und $\delta=13 \%$. Die entsprechenden Messungen wurden nach DIN 50133 (Härte) und DIN 50146,50144 (Zugfestigkeit) mit Zugproben B 10·100 nach DIN 50125 durchgeführt.

Bei der Untersuchung der Proben aus dem bestrahlten Brennelement bestand keine Möglichkeit zur Anfertigung von Zugproben. Daher mußte die mechanische Werkstoffprüfung auf Härtemessungen beschränkt werden. Diese brachten für die Randzone und die Zentralzone des Brennstoffstabes in den verschiedenen Probeentnahmebereichen (Bild 2) die in Tabelle 1 zusammengestellten Ergebnisse.

Nach den Ergebnissen von Tabelle 1 war die Härte in der Randzone des Brennstoffstabes bei niedrigen Bestrahlungstemperaturen nach etwa 0,1\% Abbrand des Urans um 150 Vickershärteeinheiten gestiegen. Die nur wenig niedriger liegende Härte nach $0,065 \%$ Abbrand in der Randzone des Abschnitts 6 deutet darauf hin, daß die Härtesteigerung durch Bestrahlung bei diesem $\mathrm{Ab}$ brand praktisch schon ihre Sättigung erreicht hat. In der Zentralzone des Brennstoffstabes ist dagegen die

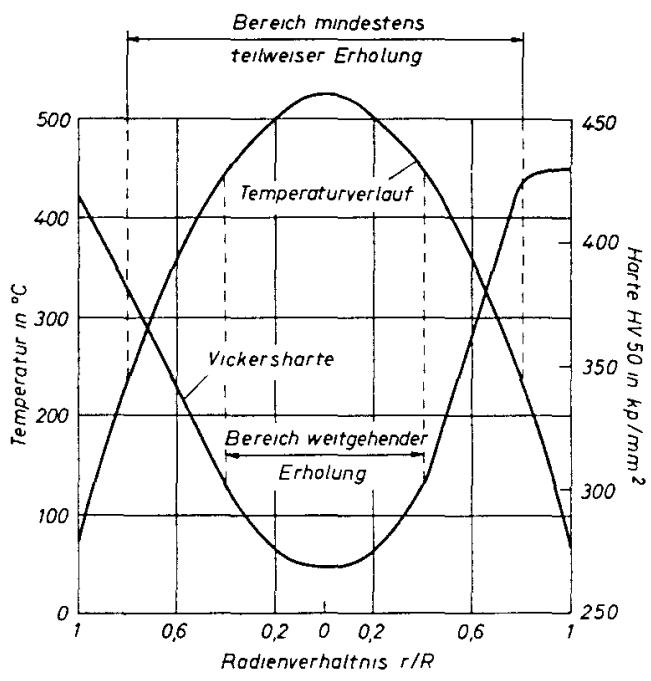

Bild 10. Verlauf der Härte und der maximalen Bestrahlungstemperatur längs eines Brennstoffstabdurchmessers im $\mathrm{Ab}$ schnitt 4 des bestrahlten Brennelementes

Härte bei den relativ hohen Bestrahlungstemperaturen von 440 bis $530^{\circ} \mathrm{C}$ (Abschnitte 1 bis 5) unverändert geblieben. Bei diesen Temperaturen findet, wie schon die oben dargestellten Gefügeuntersuchungen zeigten, eine nahezu vollständige Erholung der Gefügeschäden während der Bestrahlung statt. Bei einer Bestrahlungstemperatur von $300^{\circ} \mathrm{C}$ (Zentralzone des Abschnittes 6) ergibt sich nur noch eine teilweise Erholung, die die Härtesteigerung bei etwa $50 \%$ des höchstmöglichen Wertes hält.

In Bild 10 ist der Verlauf der Härte und der maximalen Bestrahlungstemperatur längs eines Brennstoff- 
stabdurchmessers im Abschnitt 4 wiedergegeben. Nach dieser Darstellung erscheint es sinnvoll, oberhalb von $450{ }^{\circ} \mathrm{C}$ mit weitgehender Erholung, unterhalb von $250{ }^{\circ} \mathrm{C}$ dagegen mit nur unwesentlicher Erholung der Gefügeschäden während der Bestrahlung zu rechnen. Diesen Annahmen entsprechen auch die im vorangegangenen Abschnitt dargestellten Ergebnisse. Allerdings müssen die hier gezogenen Schlüsse auf den $\mathrm{Zu}$ sammenhang mit den Neutronenfluß- und Abbrandwerten in den untersuchten Brennstoffprobenbereichen beschränkt bleiben.

Verringerung der Dichte. An verschiedenen Proben aus dem unbestrahlten Brennelement wurde die Dichte des mit etwa $0,4 \mathrm{Gew} .-\%$ Nb legierten Uranstabmaterials bei $20^{\circ} \mathrm{C}$ im Mittel mit $\varrho=(18,90 \pm 0,02) \mathrm{g} / \mathrm{cm}^{3}$ gefunden. Gelegentlich ergaben sich weit aus dem angegebenen Schwankungs. bereich herausfallende Werte, die wahrscheinlich durch die relativ zahlreichen Lunker im Brennstoffstab verfälscht waren. Aus den Probenentnahmebereichen des bestrahlten Brennelementes (Bild 2) wurden zur Dichtebestimmung etwa $2 \mathrm{~cm}^{3}$ große Proben herausgeschnitten, die den zentralen Bereich oder einen Anteil der äußeren Zone des Brennstoffstabes erfaßten. Diese Proben wiesen bei $20^{\circ} \mathrm{C}$ die in Tabelle 2 zusammengestellten Dichten auf.

Das Ergebnis ist für die Randzone völlig eindeutig: Die Dichteverringerung beträgt durchschnittlich mit relativ geringer Schwankung $0,3 \%$ je $0,1 \%$ Abbrand und ergibt sich fast vollständig aus der Einlagerung der Spaltproduktatome im Uran, deren Anteil auf $0,25 \%$ je $0,1 \%$ Abbrand geschätzt wird ${ }^{5}$ ). Korngrenzendefekte durch Versprödung und Temperaturwechsel, deren Beitrag zur Dichteverringerung bis zu $0,3 \%$ je $0,1 \%$ Abbrand betragen kann ${ }^{5}$ ), können also nur in geringer Zahl und Ausdehnung entstanden sein. Das ist auf die relativ niedrige Bestrahlungstemperatur unterhalb $400{ }^{\circ} \mathrm{C}$ im Bereich der Proben aus der Randzone des Brennstoffstabes zurückzuführen.

Für die größeren Dichteverringerungen von 0,6 bis $0,8 \%$ je $0,1 \%$ Abbrand, die an den Proben aus der Zentralzone gemessen wurden, könnten über die Einlagerung von Spaltproduktatomen hinaus grundsätzlich Korngrenzendefekte und Schwellung des Urans durch anisotrope thermische Ausdehnung bzw. durch Ausscheidung von Spaltgasporen verantwortlich gemacht werden. Die entsprechenden Mechanismen, die bei Bestrahlungstemperaturen oberhalb $400{ }^{\circ} \mathrm{C}$ in Tätigkeit treten, dürften jedoch durch den mechanischen Widerstand der kälteren Randzone des Brennstoffstabes behindert worden sein. Auch die besonders hohe Dichteverringerung von etwa $0,8 \%$ je $0,1 \%$ Abbrand bei der relativ niedrigen Zentraltemperatur von $300^{\circ} \mathrm{C}$ im $\mathrm{Ab}$ schnitt 6 spricht gegen die Deutung durch direkte Einwirkung von Korngrenzendefekten und Schwellung. Als primäre Ursache wird vielmehr eine bleibende Aufweitung der Randzone des Brennstoffstabes durch Kriechverformung unter dem Einfluß thermischer Spannungen angesehen, die durch das steile radiale Temperaturgefälle hervorgerufen werden. Diese Aufweitung führt bei Abkühlung des Brennstoffstabes zu dichteverringernden Gefügedefekten in der Zentralzone, deren Ausmaß nicht vom Abbrand, sondern von der Größe des radialen Temperaturgefälles im Brennstoffstab abhängt.
Änderung der Leitfähigkeit. Bei den vorbereitenden Messungen an zahlreichen Querschnitten des unbestrahlten Brennelementes ergaben sich elektrische Leitfähigkeitswerte des Uranbrennstoffes von $\sigma=(3,18 \pm 0,03) \mathrm{m} / \Omega \mathrm{mm}^{2}$. Die in verschiedenen Zonen eines Querschnittes gemessenen Leitfähigkeitswerte wichen um höchstens $0,01 \mathrm{~m} / \Omega \mathrm{mm}^{2}$ voneinander $\mathrm{ab}$. Die Messungen an Querschnitten des bestrahlten Brennelementes zeigten, daß durch die Bestrahlung eine nur geringfügige Änderung der elektrischen Leitfähigkeit eingetreten war (s. Tabelle 3). Die elektrische Leitfähigkeit war also um höchstens $1 \%$ gesunken und folgte damit der Abschätzung von A. D. B r a il s f ord und K. G. $\mathrm{Maj}$ o ${ }^{16}$ ), die für die eingehaltenen Bestrahlungsbedingungen eine Leitfähigkeitsminderung um 0,8 bis $1,2 \%$ erwarten ließ. Die Tatsache, daß die Leitfähigkeitswerte in der Zentralzone des Brennstoffstabes deutlich niedriger lagen als in der Randzone, läßt sich durch eine Porosität von etwa $0,5 \%$ in der Zentralzone erklären. Diese Deutung entspricht vollständig den Ergebnissen der Dichtebestimmung und ist auch im gleichen Sinn zu erläutern.

$$
\begin{gathered}
\text { Preßsitzverbindungen der } \\
\text { Brennstoffstabteile }
\end{gathered}
$$

Aufbeulung im Preßsitzbereich. Wiebereits oben vermerkt worden ist, zeigte das bestrahlte Brennelement im Bereich der Preßsitzverbindungen zwischen den drei Brennstoffstabteilen während der Bestrahlung ein anderes Verhalten als in ungestörten Be. reichen des Brennstoffstabes. Den ersten entsprechenden Hinweis gaben deutlich sichtbare Aufbeulungen der Brennstoffhülle. Schon die Probenentnahme brachte weitgehend Aufschluß über den Charakter des vermuteten Schadens. Ein Querschnitt durch den unteren Preßsitz ließ erkennen, daß sich zwischen Zapfen und Fassung des Preßsitzes ein etwa $1 \mathrm{~mm}$ breiter Ringspalt gebildet hatte (Bild 11). Radialschnitte zeigten außerdem einen umlaufenden konischen Riß im Ubergang zwischen der ringförmigen Fassung des Preßsitzes und dem vollen Brennstoffstab (Bild 1). Die aus dem Bereich beider Preßsitze entnommenen Proben zerfielen gemäß der Unterteilung durch diesen Riß und den Ringspalt.

Das $V$ orhandensein des breiten Ringspaltes beweist, daß die Aufweitung der ringförmigen Preßsitzfassungen um etwa $2 \mathrm{~mm}$ nicht durch Formänderungen des Preßsitzzapfens verursacht worden ist. Der Mechanismus des Aufweitungsvorganges dürfte vielmehr gerade auf der Unterbrechung des Materialzusammenhanges durch die Bohrung des Preßsitzes beruhen. Durch diese wird ein Uranring mit dem steilen radialen Temperaturgefälle der Randzone des Brennstoffstabes während der Bestrahlung vom mechanischen Widerstand des zentralen Stabbereiches gegen radiale Aufweitung entlastet.

Mit dem radialen Temperaturgefälle bauen sich in dem Uranring außen Zug- und innen Druckspannungen auf, die aufgrund der niedrigen Kriechfestigkeit des Urans teilweise durch Verformung abgebaut werden. Beim Wegfall des Temperaturgefälles tritt eine Spannungsumkehr ein, die infolge des vorangegangenen Spannungsabbaues zu gegenläufigen Verformungen führt. Unsymmetrien der Zug-Druck-Spannungsfolge führen zu einer Begünstigung der Zugverformung und damit zu einer bleibenden Aufweitung des Uranringes nach jedem Temperaturwechsel. Ein entsprechender 
Mechanismus ist vor allem im Zusammenhang mit der Verformung von Druckbehälterwänden als "thermal ratcheting" geläufig. Unter Bestrahlung ergibt sich zusätzlich eine bedeutende Erhöhung der Kriechgeschwindigkeit des Urans, u. U. um 1 bis 2 Größenordnungen ${ }^{18}$ ) .

Durch die zahlreichen Temperaturwechsel beim Anfahren und Abschalten des Reaktors hat sich eine stufen-

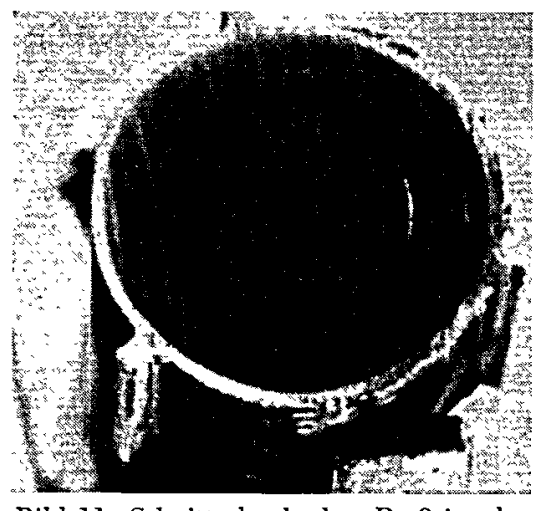

Bild 11. Schnitt durch den Preßsitz des bestrahlten Brennelementes

weise Aufweitung der Preßsitzfassungen bis zu beträchtlichen Ausmaßen ergeben, die schließlich im Zusammenhang mit der Kerbwirkung am Boden der Preßsitzbohrung und der Versprödung des Urans durch die Bestrahlung zur Bildung des erwähnten umlaufenden Risses im Ubergang zum vollen Brennstoffstab führte. tur (sie betrug im Abschnitt 5 normalerweise höchstens $440^{\circ} \mathrm{C}$ ) erklärt sich aus dem Verlust des Wärmekontakts zwischen Zapfen und Fassung des Preßsitzes bei Bildung des beobachteten Ringspaltes. Die Spaltungswärme konnte dadurch aus dem Zapfen im wesentlichen nur noch in axialer Richtung abgeführt werden. Selbst unter der Annahme, daß an der Stirnfläche des Zapfens durch thermische Ausdehnung (auch der gegenüberliegenden Zentralzone des vollen Brennstoffstabes) und Schwellung des Urans durch Spaltgasausscheidung (s. u.) ein guter Wärmekontakt hergestellt war, ergibt eine überschlägige Rechnung, daß in der Umgebung der mittleren Querschnittsebenen der Zapfen Temperaturen von etwa $570^{\circ} \mathrm{C}$ im oberen Preßsitz und von etwa $680^{\circ} \mathrm{C}$ im unteren Preßsitz erreicht worden sein müssen. Bei vollständigem Wegfall des Wärmekontaktes an der Zapfenstirnfläche könnten in der Umgebung der Stirnfläche sogar Temperaturen von 1100 bis $1200{ }^{\circ} \mathrm{C}$ erreicht werden, so daß u. U. der Schmelzpunkt des Urans (etwa $1130^{\circ} \mathrm{C}$ ) überschritten würde.

Die beobachteten Gefügeänderungen entsprechen allerdings der ersten Annahme, nach der das Uran der Preßsitzzapfen während der Bestrahlung fast ausnahmslos in der $\alpha$-Phase vorgelegen hat $(\alpha-\beta$-Umwandlungstemperatur etwa $665^{\circ} \mathrm{C}$ ). In der $\beta$. Phase wären wesentlich stärkere Kornvergröberungen aufgetreten. Neben der bereits erwähnten starken Rekristallisation sind Rißbildung an Korn- und Zwillingsgrenzen (Bild 13) und Schwellung durch Spaltgasporen (Bild 14) festzustellen.

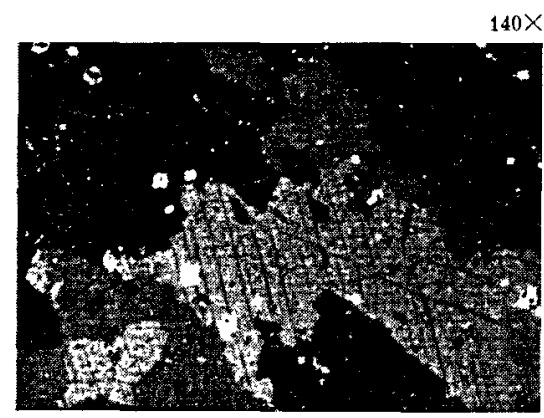

Bild 12. Gefüge des bestrahlten Brennelementes, Schliff parallel zur Preßrichtung. Zapfen des oberen Preßsitzes (polarisiertes Licht)

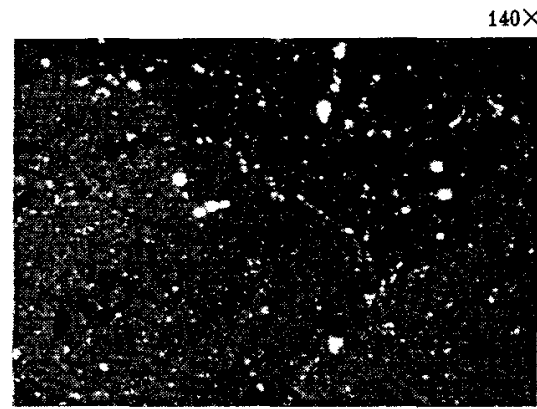

Bild 13. Gefüge des bestrahlten Brennelementes, Schliff quer zur Preßrichtung. Intergranulare Risse im Zapfen des oberen Preßsitzes (polarisiertes Licht)

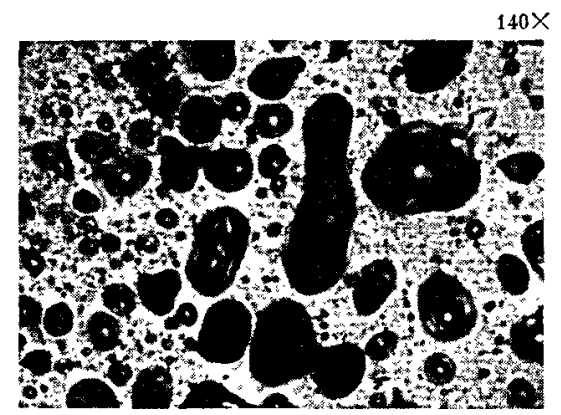

Bild 14. Gefüge des bestrahlten Brennelementes, Schliff quer zur Preßrichtung. Poren in der Kernzone des Zapfens des unteren Preßsitzes (Hellfeld)
Gefügeschädigung. Die besonderen Eigenschaftsänderungen des bestrahlten Uranbrennstoffes im Bereich der Preßsitze ergeben sich aus dem oben beschriebenen Schaden des Brennstoffstabes. Das Urangefüge in den ringförmigen Fassungen der Preßsitze bot im wesentlichen das gleiche Bild wie in der Randzone anderer Bereiche des Brennstoffstabes. Die Gefügeänderungen durch die relativ geringe Verformung um höchstens etwa $6 \%$ bei der Aufweitung der Preßsitzfassungen gehen in der sehr starken Gefügeschädigung durch die Bestrahlungseffekte unter.

Dagegen wich das Gefüge in den Zapfen beider Preßsitze erheblich von dem im sonstigen zentralen Bereich des Brennstoffstabes ab. Es war in groben Körnern rekristallisiert (Bild 12), die die Korngröße des unbestrahlten Gefüges so weit überschritten, daß in den Zapfen eine oberhalb der Rekristallisationstemperatur von etwa $550^{\circ} \mathrm{C}$ liegende Bestrahlungstemperatur geherrscht haben muß. Die Erhöhung der Zentraltempera-

18) A. S. Z a im ow s k et al., Proc. Second Geneva Conf. (1958). UN, Genf 5 (1958) P/2191, S. 566.
Nach Angaben von R. G. B ell a m y ${ }^{10}$ ) sind Mikrorisse in $\alpha$-Uran bei Bestrahlung und Temperaturwechseln mit Temperaturen über $450^{\circ} \mathrm{C} z u$ erwarten. Die ursächlichen Spannungen zwischen den Körnern des Urangefüges ergeben sich durch die stark anisotrope thermische Ausdehnung des Urans. Aufgrund der schon nach niedrigem Abbrand des Urans von etwa $0,02 \%$ fast vollständigen Versprödung bilden sich Mikrorisse durch Sprödbrüche an Korngrenzen bei hohen Temperaturen von etwa $600{ }^{\circ} \mathrm{C}$, in deren Bereich die Korngrenzenfestigkeit schnell abnimmt. Transgranulare Sprödbrüche an Zwillingsgrenzen werden dagegen bei relativ tiefen Temperaturen von hohen Spannungen nach Abkühlung des Urans durch ein breites Temperaturintervall ausgelöst.

Die Schwellung des Urans durch die Ausscheidung und das Wachsen von Spaltgasporen setzt bei $400^{\circ} \mathrm{C}$ ein und wird durch Temperaturanstieg oberhalb von $500{ }^{\circ} \mathrm{C}$ stark beschleunigt $\left.\left.{ }^{2}\right)^{5}\right)^{6}$ ). Das ungehemmte Wirken dieses Prozesses in den Preßsitzzapfen des bestrahlten Brennelementes wurde durch die Bildung der Ring- 
spalte in den Preßsitzen ermöglicht, die die Zapfen vom mechanischen Widerstand der Fassungen gegen radiale Ausdehnung entlastete.

Änderung der Härte, Dichte und elektrischen Leitfähigkeit. Durch die schwache Verformung bei der Aufweitung der ringförmigen Preßsitzfassungen wurde die Härte, wie auch das Gefüge, nicht nachweisbar verändert. Die Härtemessungen an Proben aus beiden Preßsitzfassungen ergaben Werte von $H V 50=(420 \pm 20) \mathrm{kp} / \mathrm{mm}^{2}$. Diese stimmen innerhalb der Schwankungsgrenzen mit den oben angegebenen Härtewerten für die Randzone in ungestörten Brennstoffstabbereichen überein.

Die in den Preßsitzzapfen gemessenen Härtewerte zeigten weite, wenig systematische Schwankungen, die der vielfältigen Form der Urangefügeausbildung entsprachen. Ohne auf die wenig aufschlußreiche räumliche Verteilung der Härtewerte in den Zapfen im einzelnen einzugehen, kann doch zusammenfassend festgestellt werden, da $\beta$ in den Bereichen, in denen das Kornwachstum des Urans behindert war, die Härte HV50 $=(290 \pm 10)$ $\mathrm{kp} / \mathrm{mm}^{2}$ etwa mit den in der Zentralzone ungestörter Brennstoffstabbereiche gemessenen Werte übereinstimmte, während die von der Grobkornbildung erfaßten Gefügeanteile eine erhebliche Härteminderung auf HV50 $=(215 \pm 20) \mathrm{kp} / \mathrm{mm}^{2}$ erlitten hatten.

Dichtebestimmungen an zwei Proben, die jeweils den größten Teil eines Preßsitzzapfens erfaßten, ergaben Dichten von 17,357 und $18,264 \mathrm{~g} / \mathrm{cm}^{3}$ für den unteren bzw. oberen Preßsitz. Diese Werte entsprechen Dichteverringerungen von $6,8 \%$ je $0,1 \%$ Abbrand und $3,4 \%$ je $0,1 \%$ Abbrand und gaben damit einen neuen Beweis für die starke Schwellung des Urans in den Preßsitzzapfen infolge der bereits in Gefügebildern nachgewiesenen ausgedehnten Spaltgasporenbildung. Die Dichteverringerung im oberen Preßsitzzapfen stimmt mit Literaturangaben über Bestrahlungsversuche bei etwa $500^{\circ} \mathrm{C}$ überein $\left.\left.{ }^{2}\right)^{6}\right)^{7}$ ). Der hohe Wert für den unteren Preßsitzzapfen kann sich nur am oberen Ende des $\alpha$-Phasentemperaturgebietes weit oberhalb der Rekristallisationstemperatur, d.h. also oberhalb $600^{\circ} \mathrm{C}$ ergeben haben ${ }^{7}$ ). Dieses Ergebnis stimmt mit den oben gezogenen Schlüssen bezüglich der Bestrahlungstemperatur der Preßsitzzapfen überein. An den gleichen Proben aus beiden Preßsitzzapfen wurden elektrische Leitfähigkeiten von 2,48 für den unteren und $2,70 \mathrm{~m} / \Omega$ $\mathrm{mm}^{2}$ für den oberen Preßsitz gemessen. Die Leitfähigkeitsminderung gegenüber dem unbestrahlten Uranbrennstoff betrug also $22 \mathrm{bzw} .15 \%$.

\section{Zusammenfassung}

Aus einem aus metallischem Uran bestehenden FR2Brennstoffstab wurden für Werkstoffuntersuchungen Proben entnommen, die mit thermischen Neutronenflüssen von 1,8 bis $3,2 \cdot 10^{13} \mathrm{n} / \mathrm{cm}^{2} \mathrm{~s}$ bis zu Uranabbränden von 0,06 bis $0,12 \%$ bestrahlt worden waren. An diesen Proben wurden mikroskopische Untersuchungen sowie Messungen der Härte, Dichte und elektrischen Leitfähigkeit durchgeführt.

Bei Bestrahlungstemperaturen unterhalb von $250{ }^{\circ} \mathrm{C}$ wird das Urangefüge des Brennstoffstabes vollständig zerstört. Die kristallographische Ordnung bleibt dabei aufrechterhalten. Das Gefüge entspricht etwa $\operatorname{dem} \mathrm{Zu}$ stand nach sehr starker Verformung. Die Härte steigt um 130 bis 150 Vickershärteeinheiten. Die Dichteverringerung hält sich mit $0,3 \%$ je $0,1 \%$ Abbrand im
Rahmen der Auswirkung der Spaltprodukteinlagerung. Dasselbe gilt für die Verringerung der elektrischen Leitfähigkeit, die höchstens $1 \%$ beträgt. Rißförmige $\mathrm{Ge}$ fügedefekte konnten nicht nachgewiesen werden.

Bei Bestrahlungstemperaturen oberhalb von $250^{\circ} \mathrm{C}$ tritt eine teilweise, oberhalb von $450{ }^{\circ} \mathrm{C}$ eine weitgehende Erholung der Gefügeschäden und damit auch der Härteänderung ein. Korngrenzendefekte oder Spaltgasporen konnten im Zentralbereich des vollen Brennstoffstabes nicht direkt beobachtet werden, obwohl die Verringerung der Dichte und elektrischen Leitfähigkeit auf eine Porosität von etwa $0,4 \%$ schließen läßt.

Zusammenfassend kann festgestellt werden, $\mathrm{da} ß$ die beobachteten Eigenschaftsänderungen des Urans während der Bestrahlung die Verwendungsfähigkeit des Brennstoffstabes nicht in Frage stellen. Eine nennenswerte Verschlechterung des Materialzusammenhanges (durch Risse oder Poren) war nicht eingetreten. Die bleibende Vergrößerung des Stabdurchmessers (entsprechend Messungen der Dichte) betrug höchstens $0,2 \%$.

Als kritische Bereiche haben sich die Preßsitze zwischen den drei Teilstücken des Brennstoffstabes erwiesen. Die ringförmigen Preßsitzfassungen sind infolge betriebsbedingter Temperaturwechsel schrittweise aufgeweitet worden (hier um maximal $6 \%$ ). Durch Unterbrechung der radialen Wärmeableitung ist es zu einer Überhitzung der Preßsitzzapfen gekommen, die die Bildung zahlreicher Risse im Urangefüge, starke Schwellung durch Spaltgasporenausscheidung und eventuell auch Phasenumwandlungen verursacht hat.

Die Herren F. Mertgen und W. Stegmaier waren am Aufbau der Versuchseinrichtung maßgeblich beteiligt. An der Untersuchung des Brennelementes haben die Herren L. R. Glahn, Dipl.-Ing. H.J. $\mathrm{H} \mathrm{a} \mathrm{u} \mathrm{ck,} \mathrm{H.} \mathrm{Lei} \mathrm{er} \mathrm{und} \mathrm{F.} \mathrm{Mer} \mathrm{t} \mathrm{g} \mathrm{e} \mathrm{n} \mathrm{mitgearbeitet.}$ Herr Dr. P. $Z$ ü hl k e führte Vorarbeiten für die Auswertung des Bestrahlungsversuches durch.

\section{Summary}

Investigation of an Irradiated FR2. fuel Element. Samples were taken from a FR2fuel rod which had been irradiated with thermal neutron fluxes of 1.8 to $3.2 \cdot 10^{13} \mathrm{n} / \mathrm{cm}^{2} \cdot \mathrm{s}$ up to uranium burnup of 0.06 to $0.12 \%$. Microstructure, hardness, density and electrical conductivity were measured.

Below $250^{\circ}$ the uranium microstructure is completely destroyed by the irradiation, while the crystal structure is preserved. The microstructure is similar to that after heavy deformation. The hardness increases by 130 to 150 DPH. Density decreases by $0.3 \%$ per $0.1 \%$ uranium burnup, electrical conductivity decreases by less than $1 \%$. Cracks were not found. Above $250^{\circ}$ partial recovery occurs which is complete above $450^{\circ} \mathrm{C}$. Grain boundary defects or pores due to fission gas could not be found in the central area of the fuel rod, althaugh the decrease of density and electrical conductivity points to a porosity of about $0.4 \%$.

The investigated changes of properties indicate that these fuel rods are suited for in-pile use, the critical areas being localized at the ring-plug connexions of the three fuel rod parts, where overheating, and consequently crack formation, precipitation of gases and possibly phase transformation were found.

(Eingegangen am 21. November 1961) 